Alper Atamtürk · George L. Nemhauser · Martin W.P. Savelsbergh

\title{
Valid inequalities for problems with additive variable upper bounds ${ }^{\star}$
}

Received: April 15, 1999 / Accepted: October 10, 2000

Published online May 18, 2001 - (C) Springer-Verlag 2001

\begin{abstract}
We study the facial structure of a polyhedron associated with the single node relaxation of network flow problems with additive variable upper bounds. This type of structure arises, for example, in production planning problems with setup times and in network certain expansion problems. We derive several classes of valid inequalities for this polyhedron and give conditions under which they are facet-defining. Our computational experience with large network expansion problems indicates that these inequalities are very effective in improving the quality of the linear programming relaxations.
\end{abstract}

\section{Introduction}

We study a polyhedron that arises as a relaxation of network flow problems with additive variable upper bounds, such as network expansion problems and production planning problems with setup times. In a network expansion problem, given a network, with demands on the nodes and capacities on the arcs, we are interested in installing additional capacities on subsets of the arcs of the network so that the total cost of additional capacity installation is minimized. In practice, the capacity cost is a piecewise linear function that exhibits a strong economy of scale. Here we are interested in certain capacity installation cost functions that can be modeled using binary variables, such as step function costs, see $[1,4,14]$. Mixed-integer programming formulations of such network expansion problems typically include constraints of the following type (and possibly other side constraints) for each node of the network

$$
\begin{aligned}
& \sum_{i \in M^{+}} y_{i}-\sum_{i \in M^{-}} y_{i} \leq b \\
& y_{i} \leq u_{i}+\sum_{j \in N(i)} a_{i j} x_{j}, i \in M \\
& y_{i} \in \mathbb{R}_{+}, x_{j} \in\{0,1\}, j \in N(i), i \in M .
\end{aligned}
$$

Inequality (1) is the balance constraint of a node with inflow $\left(M^{+}\right)$and outflow $\left(M^{-}\right)$ edges and demand $b$. The continuous variable $y_{i}$ represents the flow on edge $i, i \in M=$ $M^{+} \cup M^{-}$. Inequalities (2) are the additive variable upper bound (AVUB) constraints

A. Atamtürk: Department of Industrial Engineering and Operations Research, University of California, Berkeley, CA 94720-1777, USA, e-mail: atamturk@ieor. berkeley . edu

G.L. Nemhauser, M.W.P. Savelsbergh: School of Industrial and Systems Engineering, Georgia Institute of Technology, Atlanta, GA 30332-0205, USA, e-mail: \{gnemhaus \} \{mwps \} @isye.gatech . edu

* This research is supported, in part, by NSF Grant DMI-9700285 to the Georgia Institute of Technology. 
on the flow variables. $N(i)$ is the index set of binary variables $x_{j}$ representing the availability of resources that increase the capacity of edge $i$ that has capacity $u_{i}$. Step function capacity installation costs can be modeled with inequality (2) and generalized upper bound constraints $\sum_{j \in N(i)} x_{j} \leq 1$.

Multi-item production planning problems with setup times have the following constraints as part of their MIP formulations

$$
\begin{array}{ll}
\sum_{t^{\prime} \leq t} y_{i t^{\prime}} \geq d_{i t}, & \forall i, t \\
\sum_{i} y_{i t} \leq u_{t}-\sum_{i} a_{i} x_{i t}, \quad \forall t
\end{array}
$$

where $d_{i t}$ denotes the demand for item $i$ in period $t, u_{t}$ the total production capacity in period $t$ and $a_{i}$ the setup time required for item $i$ if the machine is setup for this item. Aggregating the demand constraints (4) and the production variables $y_{i t}$ for each period, we arrive at the same structure as in (1)-(2).

Additive variable upper bounds (2) generalize simple variable upper bounds $y_{i} \leq$ $u_{i} x_{i}$ in three respects. First, several binary variables additively increase the upper bound on the continuous variable. Second, the continuous variable is not necessarily restricted to zero when its additive variable bounds are zero. Third, we allow an overlap of additive variable upper bound variables, i.e., $N(i) \cap N(k) \neq \emptyset$ for $i, k \in M$. This situation typically occurs when capacities are installed on subsets of edges such as on cycles of the network (rings). We also point out that a variable lower bound constraint $l_{i} x_{i} \leq y_{i}$ can be put into a simple form of AVUB, $\bar{y}_{i} \leq\left(u_{i}-l_{i}\right)+l_{i} \bar{x}_{i}$, after complementing the binary variable $x_{i}$ and the continuous variable $y_{i}$ assuming that it has a finite upper bound $u_{i}$. Therefore, the polyhedron with additive variable upper bounds generalizes the single node fixed-charge flow polyhedron introduced in $[12,15]$.

In the next section we introduce four classes of valid inequalities for

$$
P=\left\{(x, y) \in\{0,1\}^{n} \times \mathbb{R}_{+}^{m}: \text { subject to (1) and (2) }\right\}
$$

and give conditions under which these inequalities are facet-defining for $\operatorname{conv}(P)$. In Sect. 3 we discuss how to find inequalities that are violated by linear programming solutions. In Sect. 4 we present a summary of computational results on the use of the new inequalities in a branch-and-cut algorithm for network expansion problems.

\section{Valid inequalities}

Let $N=\{1,2, \ldots, n\}$ be the index set of binary variables and $M=\{1,2, \ldots, m\}$ be the index set AVUB constraints. Let $N(i)$ be the subset of $N$ appearing in the AVUB constraint indexed $i$ for $i \in M$ and $M(j)$ be the index set of AVUB constraints that $x_{j}$ appears for $j \in N$. Let $N(S)=\cup_{i \in S} N(i)$ for $S \subseteq M$. We define $u(S)=$ $\sum_{i \in S}\left(u_{i}+\sum_{j \in N(i)} a_{i j}\right)$ for $S \subseteq M$ and $a_{j}(S)=\sum_{i \in S} a_{i j}$ for $j \in N(S)$. So,

$$
\sum_{i \in S} y_{i} \leq \sum_{i \in S}\left(u_{i}+\sum_{j \in N(S)} a_{j}(S) x_{j}\right) \leq u(S) \text { for } S \subseteq M .
$$


For notational simplicity we use $u(i)$ for $u(\{i\})$. Throughout we make the following assumptions on the data of the model (1)-(3).

Assumptions:

(A.1) $a_{i j}>0$ for all $i \in M, j \in N(i)$.

(A.2) $u(i)>0$ for all $i \in M$.

(A.3) $b+u\left(M^{-}\right)>0$.

(A.4) $u(i)-a_{i j} \geq 0$ for all $i \in M, j \in N(i)$.

(A.5) $b+u\left(M^{-}\right)-a_{j}\left(M^{-}\right) \geq 0$ for all $j \in N$.

Assumptions (A.2)-(A.5) are made without loss of generality. If $u(i)<0$ or $b+$ $u\left(M^{-}\right)<0$, then $P=\emptyset$. If $u(i)=0\left(b+u\left(M^{-}\right)=0\right)$, then $y_{i}=0\left(y_{i}=0, i \in\right.$ $\left.M^{+}\right)$in every feasible solution and can be eliminated. Similarly if $u(i)-a_{i j}<0$ or $b+u\left(M^{-}\right)-a_{j}\left(M^{-}\right)<0$, then $x_{j}=1$ in every feasible solution and can be eliminated. Note that given (A.1), if $N(i) \neq \varnothing$ for all $i \in M$, then (A.4) implies (A.2) and (A.5) implies (A.3). Assumption (A.1) is made for convenience. Results presented in the sequel can easily be generalized to the case with $a_{i j}<0$. Note that, for a particular $j \in N$ if $a_{i j}<0$ for all $i \in M$, then $x_{j}$ can be complemented to satisfy (A.1). If there is no overlap of additive variable upper bounds, i.e., $N(i) \cap N(k)=\emptyset$ for all $i, k \in M$, then $M(j)$ is singleton for all $j \in N$ and (A.1) can be satisfied by complementing the binary variables when $a_{i j}<0$.

Proposition 1. [2] Conv $(P)$ is full-dimensional.

\subsection{Additive flow cover inequalities}

Let $C^{+} \subseteq M^{+}, C^{-} \subseteq M^{-}, L^{-} \subseteq M^{-} \backslash C^{-}$and $K=M^{-} \backslash\left(C^{-} \cup L^{-}\right)$. By relaxing inequality (1) using $y_{i} \geq 0, i \in M^{+} \backslash C^{+}, y_{i} \leq u(i), i \in C^{-}$, and $y_{i} \leq u_{i}+$ $\sum_{j \in N(i)} a_{i j} x_{j}, i \in L^{-}$, we obtain the following valid inequality for $P$

$$
\sum_{i \in C^{+}} y_{i}-\sum_{j \in N\left(L^{-}\right)} a_{j}\left(L^{-}\right) x_{j}-\sum_{i \in K} y_{i} \leq b+u\left(C^{-}\right)+\sum_{i \in L^{-}} u_{i} .
$$

The inequality we will present next is a strengthening of (6). For $C^{+} \subseteq M^{+}$and $C^{-} \subseteq M^{-},\left(C^{+}, C^{-}\right)$is said to be a flow cover if $\lambda=u\left(C^{+}\right)-b-u\left(C^{-}\right)>0$. For a flow cover $\left(C^{+}, C^{-}\right)$, let $L^{-} \subseteq M^{-} \backslash C^{-}$be such that $\gamma=\sum_{i \in L^{-}} u_{i}<\lambda$ and $K=M^{-} \backslash\left(C^{-} \cup L^{-}\right)$. Then, the additive flow cover inequality is

$$
\begin{aligned}
\sum_{i \in C^{+}} y_{i}+ & \sum_{j \in N\left(C^{+}\right)}\left(a_{j}\left(C^{+}\right)-\lambda+\gamma\right)^{+}\left(1-x_{j}\right)- \\
& \sum_{j \in N\left(L^{-}\right)} \min \left\{a_{j}\left(L^{-}\right), \lambda-\gamma\right\} x_{j}-\sum_{i \in K} y_{i} \leq b+u\left(C^{-}\right)+\gamma
\end{aligned}
$$

Proposition 2. The additive flow cover inequality (7) is valid for $P$. 
Proof. Let $(\bar{x}, \bar{y}) \in P$ and $T=\left\{j \in N: \bar{x}_{j}=0\right\}$. Also define $N\left(C^{+}\right)^{+}=\{j \in$ $\left.N\left(C^{+}\right): a_{j}\left(C^{+}\right)>\lambda-\gamma\right\}, N\left(L^{-}\right)^{+}=\left\{j \in N\left(L^{-}\right): a_{j}\left(L^{-}\right)>\lambda-\gamma\right\}$, and $N\left(L^{-}\right)^{-}=N\left(L^{-}\right) \backslash N\left(L^{-}\right)^{+}$. For $(\bar{x}, \bar{y})$ the left-hand side of $(7)$, lhs, equals

$$
\sum_{i \in C^{+}} \bar{y}_{i}+\sum_{j \in N\left(C^{+}\right) \cap T}\left(a_{j}\left(C^{+}\right)-\lambda+\gamma\right)^{+}-\sum_{j \in N\left(L^{-}\right) \backslash T} \min \left\{a_{j}\left(L^{-}\right), \lambda-\gamma\right\}-\sum_{i \in K} \bar{y}_{i} .
$$

If $\left(N\left(C^{+}\right)^{+} \cap T\right) \cup\left(N\left(L^{-}\right)^{+} \backslash T\right)=\varnothing$, then

$$
l h s \leq \sum_{i \in C^{+}} \bar{y}_{i}-\sum_{j \in N\left(L^{-}\right)^{-} \backslash T} a_{j}\left(L^{-}\right)-\sum_{i \in K} \bar{y}_{i} \leq b+u\left(C^{-}\right)+\gamma .
$$

To see that the second inequality is valid, observe that $\sum_{i \in C^{+}} y_{i}-\sum_{i \in L^{-}} y_{i}-$ $\sum_{i \in K} y_{i} \leq b+u\left(C^{-}\right)$is valid for $P$ and $\sum_{i \in L^{-}} y_{i} \leq u\left(L^{-}\right)-\sum_{j \in N\left(L^{-}\right)^{+}} a_{j}\left(L^{-}\right)-$ $\sum_{j \in N\left(L^{-}\right)^{-} \cap T} a_{j}\left(L^{-}\right)$is valid for $(\bar{x}, \bar{y})$ since $N\left(L^{-}\right)^{+} \subseteq T$. Adding these two inequalities and using $\gamma=u\left(L^{-}\right)-\sum_{j \in N\left(L^{-}\right)} a_{j}\left(L^{-}\right)$gives the result.

Now, suppose $\left(N\left(C^{+}\right)^{+} \cap T\right) \cup\left(N\left(L^{-}\right)^{+} \backslash T\right) \neq \emptyset$. Then

$$
\begin{aligned}
\operatorname{lhs} \leq & u\left(C^{+}\right)-\sum_{j \in N\left(C^{+}\right) \cap T} a_{j}\left(C^{+}\right)+\sum_{j \in N\left(C^{+}\right)^{+} \cap T} a_{j}\left(C^{+}\right)+ \\
& \sum_{j \in N\left(C^{+}\right)^{+} \cap T}(\gamma-\lambda)-\sum_{j \in N\left(L^{-}\right)^{+} \backslash T}(\lambda-\gamma)-\sum_{j \in N\left(L^{-}\right)^{-} \backslash T} a_{j}\left(L^{-}\right) \\
\leq & u\left(C^{+}\right)-\lambda+\gamma-(\lambda-\gamma)\left[\left|N\left(C^{+}\right)^{+} \cap T\right|+\left|N\left(L^{-}\right)^{+} \backslash T\right|-1\right] \\
\leq & b+u\left(C^{-}\right)+\gamma .
\end{aligned}
$$

Remark 1. For the single node fixed-charge flow model, where (2) is replaced with $y_{i} \leq u_{i} x_{i}$, the additive flow cover inequality reduces to the flow cover inequality [15]

$$
\sum_{i \in C^{+}} y_{i}+\sum_{i \in C^{+}}\left(u_{i}-\lambda\right)^{+}\left(1-x_{i}\right)-\sum_{i \in L^{-}} \min \left\{u_{i}, \lambda\right\} x_{i}-\sum_{i \in K} y_{i} \leq b+\sum_{i \in C^{-}} u_{i} .
$$

Observe that one can obtain flow cover inequalities (8) for $P$ after splitting the flow variables $y_{i}$ into $y_{i 0} \geq 0$ and $y_{i j} \geq 0$ for $j \in N(i)$ so that $y_{i}=y_{i 0}+\sum_{j \in N(i)} a_{i j}$ and defining variable upper bound constraints $y_{i 0} \leq x_{i 0}$ with $x_{i 0}=1$ and $y_{i j} \leq a_{i j} x_{i j}$, for $j \in N(i)$ and then substituting $y_{i}=\sum_{j \in N(i)} y_{i j}$ and $x_{i 0}=1$. However, this approach may result in weaker inequalities than additive flow cover inequalities (7) if there are overlaps between AVUB constraints, i.e., $N(i) \cap N(k) \neq \emptyset$ for $i, k \in M$ or existing capacities in AVUBs $u_{i}$ for $i \in M$ are nonzero. To see this consider $y_{1}+y_{2} \leq 6, y_{1} \leq 5 x, y_{2} \leq 5 x$. The additive flow cover inequality with $C^{+}=\{1,2\}$ is $y_{1}+y_{2} \leq 6 x$, whereas the flow cover inequality with the same $C^{+}$obtained after splitting $x$ into $x^{\prime}$ and $x^{\prime \prime}$ to introduce $y_{1} \leq 5 x^{\prime}$ and $y_{2} \leq 5 x^{\prime \prime}$ and then substituting $x^{\prime}=x^{\prime \prime}=x$ is $y_{1}+y_{2} \leq 4+2 x$. For the other situation, consider $y_{1}-y_{2} \leq 3, y_{1} \leq 5 x, y_{2} \leq 1$. The additive flow cover inequality with $C^{+}=\{1\}$ and $L^{-}=\{2\}$ is $y_{1} \leq 4 x$, whereas the flow cover inequality with the same $C^{+}$and $L^{-}$obtained after introducing the variable upper bound relaxation $y_{1} \leq 4 x^{\prime}$ and then substituting $x^{\prime}=1$ is $y_{1} \leq 3 x+1$. 
Proposition 3. [2] The additive flow cover inequality (7) is facet-defining for $\operatorname{conv}(P)$ if the following five conditions are satisfied.

1. $C^{-}=\emptyset$,

2. $\max _{j \in N\left(C^{+}\right)} a_{j}\left(C^{+}\right)>\lambda-\gamma$,

3. $a_{j}\left(L^{-}\right)>\lambda-\gamma$ for some $j \in N(i)$ for all $i \in L^{-}$with $u_{i}=0$,

4. $u_{i} \geq 0$ for all $i \in L^{-}$,

5. $N\left(L^{-}\right) \cap N\left(M \backslash L^{-}\right)=\emptyset$.

Remark 2. For the single node fixed-charge flow the first three conditions of Proposition 3 reduce to $1 . C^{-}=\emptyset, 2 . \max _{j \in C^{+}} u_{j}>\lambda, 3 . u_{j}>\lambda$ for all $j \in L$, which are the sufficient conditions for inequality (8) to be facet-defining [15]. We remark that if Condition 1 does not hold, i.e., $C^{-} \neq \emptyset$, additive flow cover inequality (7) can be strengthened with a nonzero coefficient for $x_{j}, j \in N\left(C^{-}\right)$under certain conditions (see [7] for the case of flow cover inequalities). However, we prefer not to do this strengthening here, since it destroys the superadditivity of the lifting functions that will be introduced in Sect. 2.3. Nevertheless, in many cases the inequality (7) is non-redundant when $C^{-} \neq \emptyset$. Condition 2 may not be needed to define a facet in certain cases with $L^{-} \neq \emptyset$. However, it is necessary if $L^{-}=\emptyset$. Condition 3 is necessary, because if it does not hold for some $i \in L^{-}$with $u_{i}=0$, then one obtains a dominating inequality by moving $i$ to $K$. The following example shows that if Condition 4 of Proposition 3 is not satisfied, then the additive flow cover inequality may not be facet-defining. Let $P$ be given by

$$
y_{1}-y_{2}-y_{3} \leq 1, y_{1} \leq 3 x_{1}, y_{2} \leq-1+2 x_{2}+x_{3}, y_{3} \leq 2+2 x_{3} .
$$

It is easy to check that the inequality $y_{1}+\left(1-x_{1}\right)-x_{2}-x_{3} \leq 2$ with $C^{+}=\{1\}$ and $L^{-}=\{2,3\}$ is not facet-defining. Also note that if there is no overlap of additive variable upper bounds among continuous variables, then Condition 5 is trivially satisfied.

\subsection{Additive flow packing inequalities}

Let $C^{+} \subseteq M^{+}, K \subseteq M^{-}, L^{+} \subseteq M^{+} \backslash C^{+}$. By adding inequalities $y_{i} \leq u(i), i \in C^{+}$, $y_{i} \leq u_{i}+\sum_{j \in N(i)} a_{i j} x_{j}, i \in L^{+},-y_{i} \leq 0, i \in K$, we obtain the following valid inequality for $P$

$$
\sum_{i \in C^{+} \cup L^{+}} y_{i}-\sum_{j \in N\left(L^{+}\right)} a_{j}\left(L^{+}\right) x_{j}-\sum_{i \in K} y_{i} \leq u\left(C^{+}\right)+\sum_{i \in L^{+}} u_{i} .
$$

The next inequality we give is a strengthening of (9). For $C^{+} \subseteq M^{+}$and $C^{-} \subseteq M^{-}$, $\left(C^{+}, C^{-}\right)$is said to be a flow packing if $\mu=b+u\left(C^{-}\right)-u\left(C^{+}\right)>0$. For a flow packing $\left(C^{+}, C^{-}\right)$, let $L^{+} \subseteq M^{+} \backslash C^{+}$be such that $\gamma=\sum_{i \in L^{+}} u_{i}<\mu$ and $K=M^{-} \backslash C^{-}$. Then the additive flow packing inequality is

$$
\begin{aligned}
\sum_{i \in C^{+} \cup L^{+}} y_{i}- & \sum_{j \in N\left(L^{+}\right)} \min \left\{a_{j}\left(L^{+}\right), \mu-\gamma\right\} x_{j}+ \\
& \sum_{j \in N\left(C^{-}\right)}\left(a_{j}\left(C^{-}\right)-\mu+\gamma\right)^{+}\left(1-x_{j}\right)-\sum_{i \in K} y_{i} \leq u\left(C^{+}\right)+\gamma .
\end{aligned}
$$


Proposition 4. The additive flow packing inequality (10) is valid for $P$.

Proof. Let $(\bar{x}, \bar{y}) \in P$ and $T=\left\{j \in N: \bar{x}_{j}=0\right\}$. Also let $N\left(C^{-}\right)^{+}=\{j \in$ $\left.N\left(C^{+}\right): a_{j}\left(C^{-}\right)>\mu-\gamma\right\}, N\left(L^{+}\right)^{+}=\left\{j \in N\left(C^{+}\right): a_{j}\left(L^{+}\right)>\mu-\gamma\right\}$, and $N\left(L^{+}\right)^{-}=N\left(L^{+}\right) \backslash N\left(L^{+}\right)^{+}$. For $(\bar{x}, \bar{y})$ the left-hand side of $(10)$, lhs, equals

$\sum_{i \in C^{+} \cup L^{+}} \bar{y}_{i}-\sum_{j \in N\left(L^{+}\right) \backslash T} \min \left\{a_{j}\left(L^{+}\right), \mu-\gamma\right\}+\sum_{j \in N\left(C^{-}\right)^{+} \cap T}\left(a_{j}\left(C^{-}\right)-\mu+\gamma\right)-\sum_{i \in K} \bar{y}_{i}$.

If $\left(N\left(L^{+}\right)^{+} \backslash T\right) \cup\left(N\left(C^{-}\right)^{+} \cap T\right)=\emptyset$, then

$$
\text { lhs }=\sum_{i \in C^{+} \cup L^{+}} \bar{y}_{i}-\sum_{j \in N\left(L^{+}\right)^{-} \backslash T} a_{j}\left(L^{+}\right)-\sum_{i \in K} \bar{y}_{i} \leq u\left(C^{+}\right)+\gamma .
$$

Otherwise, using $\sum_{i \in C^{+} \cup L^{+}} y_{i}-\sum_{i \in K} y_{i} \leq b+u\left(C^{-}\right)$, we have

$$
\begin{aligned}
l h s \leq & b+u\left(C^{-}\right)-\sum_{j \in N\left(C^{-}\right) \cap T} a_{j}\left(C^{-}\right)-\sum_{j \in N\left(L^{+}\right)^{+} \backslash T}(\mu-\gamma)- \\
& \sum_{j \in N\left(L^{+}\right)^{-} \backslash T} a_{j}\left(L^{+}\right)+\sum_{j \in N\left(C^{-}\right)^{+} \cap T}\left(a_{j}\left(C^{-}\right)-\mu+\gamma\right) \\
\leq & b+u\left(C^{-}\right)-\mu+\gamma-(\mu-\gamma)\left[\left|N\left(L^{+}\right)^{+} \backslash T\right|+\left|N\left(C^{-}\right)^{+} \cap T\right|-1\right] \\
\leq & u\left(C^{+}\right)+\gamma .
\end{aligned}
$$

Proposition 5. [2] The additive flow packing inequality (10) is facet-defining for $\operatorname{conv}(P)$ if the following five conditions are satisfied.

1. $C^{+}=\emptyset$,

2. $u\left(M^{-}\right)+b>a_{j}\left(C^{-}\right)>\mu-\gamma$ for some $j \in N\left(C^{-}\right)$,

3. $a_{j}\left(L^{+}\right)>\mu-\gamma$ for some $j \in N(i)$ for all $i \in L^{+}$with $u_{i}=0$,

4. $u_{i} \geq 0$ for all $i \in L^{+}$,

5. $N\left(L^{+}\right) \cap N\left(M \backslash L^{+}\right)=N\left(C^{-}\right) \cap N(K)=\emptyset$.

Remark 3. For the single node fixed-charge flow model, the additive flow packing inequality reduces to the flow packing inequality [3,13]

$$
\sum_{i \in C^{+} \cup L^{+}} y_{i}-\sum_{i \in L^{+}} \min \left\{u_{i}, \mu\right\} x_{i}+\sum_{i \in C^{-}}\left(u_{i}-\mu\right)^{+}\left(1-x_{i}\right)-\sum_{i \in K} y_{i} \leq \sum_{i \in C^{+}} u_{i} .
$$

It is also possible to obtain flow packing inequalities for $P$ using the transformation mentioned in Remark 1. However such inequalities may be weaker than the additive flow packing inequalities (10) as in the case of flow cover inequalities.

As for the additive flow cover inequalities, in many cases additive flow packing inequalities are non-redundant when $C^{+} \neq \emptyset$. Similar statements as in Remark 2 apply to the other conditions of Proposition 5 as well. 


\subsection{Generalized additive flow cover inequalities}

In order to derive more general classes of valid inequalities for $P$, we fix a subset of the binary variables to zero, derive a valid inequality for the resulting projection, and then lift this inequality with the variables that are fixed to zero. More precisely, let $F \subseteq N$ and consider the projection

$$
\begin{aligned}
P_{F}=\left\{(x, y) \in\{0,1\}^{n-|F|} \times \mathbb{R}_{+}^{m}:\right. & \sum_{i \in M^{+}} y_{i}-\sum_{i \in M^{-}} y_{i} \leq b, \\
& \left.y_{i} \leq u_{i}+\sum_{j \in N(i) \backslash F} a_{i j} x_{j}, \quad i \in M\right\}
\end{aligned}
$$

of $P$ obtained by fixing $x_{j}=0$ for all $j \in F$.

For $S \subseteq M$, let $\bar{u}(S)=\sum_{i \in S}\left(u_{i}+\sum_{j \in N(i) \backslash F} a_{i j}\right)$. Let $C^{+} \subseteq M^{+}$and $C^{-} \subseteq M^{-}$ such that $\lambda=\bar{u}\left(C^{+}\right)-b-\bar{u}\left(C^{-}\right)>0$ and $L^{-} \subseteq M^{-} \backslash C^{-}$such that $\gamma=\sum_{i \in L^{-}} u_{i}<\lambda$ and $b+\bar{u}\left(C^{-}\right)+\gamma \geq 0$. Then, from Sect. 2.1 we have the following valid additive flow cover inequality for $P_{F}$

$$
\begin{aligned}
\sum_{i \in C^{+}} y_{i}+ & \sum_{j \in N\left(C^{+}\right) \backslash F}\left(a_{j}\left(C^{+}\right)-\lambda+\gamma\right)^{+}\left(1-x_{j}\right)- \\
& \sum_{j \in N\left(L^{-}\right) \backslash F} \min \left\{a_{j}\left(L^{-}\right), \lambda-\gamma\right\} x_{j}-\sum_{i \in K} y_{i} \leq b+\bar{u}\left(C^{-}\right)+\gamma .
\end{aligned}
$$

Note that inequality (12) is not necessarily valid for $P$. Also observe that there exists a point in $P_{F}$ for which the left-hand side of the inequality equals the right-hand side. This is true because by letting $x_{j}=1$ for all $j \in N\left(C^{+}\right), x_{j}=0$ for all $j \in N\left(L^{-}\right)$and $y_{i}=0$ for all $i \in K$, we can increase $\sum_{i \in C^{+}} y_{i}$ up to $b+\bar{u}\left(C^{-}\right)+\gamma$ since $\lambda>0$ and $\gamma<\lambda$. In order to derive a generalized additive flow cover inequality for $P$, we lift (12) with the variables in $F$. One way to do this, is sequential lifting, in which variables fixed to zero are introduced to (12) one at a time iteratively. In general, lifting of (12) is sequence dependent, that is, different lifting sequences may lead to different lifting coefficients for the same variable.

Let $\pi_{x} x+\pi_{y} y \leq \pi_{o}$ be a valid inequality for $P_{F}$ with zero coefficients for variables in $F$. Suppose $\pi_{x} x+\pi_{y} y \leq \pi_{o}$ is lifted with variable $x_{l}, l \in F$ that appears in the AVUB constraints $Q \subseteq M$. Then the lifting coefficient of $x_{l}$ as a function of its coefficients in the AVUB constraints is

$$
f\left(-a_{l}(Q)\right)=\pi_{o}-\max _{(x, y) \in P_{F \backslash}, x_{l}=1}\left\{\pi_{x} x+\pi_{y} y\right\} .
$$

Lemma 1. [8,16] If the lifting function $f$ is superadditive on $\mathbb{R}_{-}$, then lifting of $\pi_{x} x+\pi_{y} y \leq \pi_{o}$ is sequence independent.

First of all observe that since $b+\bar{u}\left(C^{-}\right)+\gamma \geq 0$ and hence the left-hand side of inequality (12) is maximized when $y_{i}=0$ for all $i \in M \backslash\left(C^{+} \cup L^{-} \cup C^{-}\right)$and since inequality (12) is tight, the lifting coefficients of $x_{j}$ for $j \in F \backslash N\left(C^{+} \cup L^{-} \cup C^{-}\right)$are zero. In order to make use of Lemma 1, we lift (12) in two phases. In the first phase we 
lift the inequality with the variables in $N\left(L^{-} \cup C^{-}\right) \cap F$. Then in the second phase we lift the resulting inequality with the variables in $N\left(C^{+}\right) \cap F$. When lifting the variables in phases, for convenience, we make the following assumption:

(A.6) $\left(N\left(C^{+}\right) \cap F\right) \cap\left(N\left(L^{-} \cup C^{-}\right) \cap F\right)=\emptyset$.

Even if (A.6) is not satisfied, the lifted inequality is still valid for $P$, but it may not be facet-defining for $\operatorname{conv}(P)$.

Now, let (12) be lifted first with variable $x_{l}, l \in N\left(L^{-} \cup C^{-}\right) \cap F$. Then the lifting coefficient associated with $x_{l}$ is equal to

$$
\begin{aligned}
& f\left(-a_{l}\left(L^{-} \cup C^{-}\right)\right)=b+\bar{u}\left(C^{-}\right)+\gamma-\max _{(x, y) \in P_{F \backslash l}, x_{l}=1}\left\{\sum_{i \in C^{+}} y_{i}+\right. \\
& \left.\sum_{j \in N\left(C^{+}\right) \backslash F}\left(a_{j}\left(C^{+}\right)-\lambda+\gamma\right)^{+}\left(1-x_{j}\right)-\sum_{j \in N\left(L^{-}\right) \backslash F} \min \left\{a_{j}\left(L^{-}\right), \lambda-\gamma\right\} x_{j}-\sum_{i \in K} y_{i}\right\} .
\end{aligned}
$$

Since $b+\bar{u}\left(C^{-}\right)+\gamma \geq 0$, the lifting problem has an optimal solution such that $y_{i}=0$ for all $i \in\left(M^{+} \backslash C^{+}\right) \cup K$. Let $(\bar{x}, \bar{y})$ be such an optimal solution and let $S=\left\{j \in N\left(C^{+}\right) \backslash F: \bar{x}_{j}=0\right\}$ and $T=\left\{j \in N\left(L^{-}\right) \backslash F: \bar{x}_{j}=1\right\}$. Clearly, we may assume that $S \subseteq\left\{j \in N\left(C^{+}\right) \backslash F: a_{j}\left(C^{+}\right)>\lambda-\gamma\right\}$ and $T \subseteq\left\{j \in N\left(L^{-}\right) \backslash F: a_{j}\left(L^{-}\right)>\lambda-\gamma\right\}$; otherwise we can obtain a solution with the same or better objective value by considering a subset of $S$ or $T$ satisfying these conditions. There are two cases to consider when determining the value of $f\left(-a_{l}\left(L^{-} \cup C^{-}\right)\right)$depending on how $\sum_{i \in C^{+}} y_{i}$ is bounded in an optimal solution. We analyze $f\left(-a_{l}\left(L^{-} \cup C^{-}\right)\right)$separately for each case.

Case 1: $\bar{u}\left(C^{+}\right)-\sum_{j \in S} a_{j}\left(C^{+}\right) \leq b+\bar{u}\left(C^{-}\right)+\gamma+\sum_{j \in T} a_{j}\left(L^{-}\right)+a_{l}\left(L^{-} \cup C^{-}\right)$.

$$
\begin{aligned}
f\left(-a_{l}\left(L^{-} \cup C^{-}\right)\right)= & b+\bar{u}\left(C^{-}\right)+\gamma-\left[\bar{u}\left(C^{+}\right)-\sum_{j \in S} a_{j}\left(C^{+}\right)+\right. \\
& \left.\sum_{j \in S}\left(a_{j}\left(C^{+}\right)-\lambda+\gamma\right)-\sum_{j \in T}(\lambda-\gamma)\right] \\
= & (|S \cup T|-1)(\lambda-\gamma) .
\end{aligned}
$$

Case 2: $\bar{u}\left(C^{+}\right)-\sum_{j \in S} a_{j}\left(C^{+}\right)>b+\bar{u}\left(C^{-}\right)+\gamma+\sum_{j \in T} a_{j}\left(L^{-}\right)+a_{l}\left(L^{-} \cup C^{-}\right)$.

$$
\begin{aligned}
f\left(-a_{l}\left(L^{-} \cup C^{-}\right)\right)= & b+\bar{u}\left(C^{-}\right)+\gamma-\left[b+\bar{u}\left(C^{-}\right)+\gamma+\sum_{j \in T} a_{j}\left(L^{-}\right)+\right. \\
& \left.a_{l}\left(L^{-} \cup C^{-}\right)+\sum_{j \in S}\left(a_{j}\left(C^{+}\right)-\lambda+\gamma\right)-\sum_{j \in T}(\lambda-\gamma)\right] \\
= & |S \cup T|(\lambda-\gamma)-\sum_{j \in S} a_{j}\left(C^{+}\right)-\sum_{j \in T} a_{j}\left(L^{-}\right)-a_{l}\left(L^{-} \cup C^{-}\right) .
\end{aligned}
$$


Observe that in Case 2, i.e., if the balance constraint is tight, $S=T=\emptyset$ since $a_{l}\left(L^{-} \cup C^{-}\right) \geq 0$ and by assumption $a_{j}\left(C^{+}\right)>\lambda-\gamma$ for all $j \in S$ and $a_{j}\left(L^{-}\right)>\lambda-\gamma$ for all $j \in T$. Also in Case $1, f\left(-a_{l}\left(L^{-} \cup C^{-}\right)\right)$is minimized when $S=T=\emptyset$. Then, we conclude that $f\left(-a_{l}\left(L^{-} \cup C^{-}\right)\right)=-\min \left\{a_{l}\left(L^{-} \cup C^{-}\right), \lambda-\gamma\right\}$. It is easy to see that $f$ is superadditive on $\mathbb{R}_{-}$. So from Lemma 1 , the lifting is sequence independent, that is the lifting function $f$ remains unchanged as the projected variables in $N\left(L^{-} \cup C^{-}\right) \cap F$ are introduced to inequality (12) sequentially. Therefore,

$$
\begin{aligned}
\sum_{i \in C^{+}} y_{i}+ & \sum_{j \in N\left(C^{+}\right) \backslash F}\left(a_{j}\left(C^{+}\right)-\lambda+\gamma\right)^{+}\left(1-x_{j}\right)- \\
& \sum_{j \in N\left(L^{-} \cup C^{-}\right) \cap F} \min \left\{a_{j}\left(L^{-} \cup C^{-}\right), \lambda-\gamma\right\} x_{j}- \\
& \sum_{j \in N\left(L^{-}\right) \backslash F} \min \left\{a_{j}\left(L^{-}\right), \lambda-\gamma\right\} x_{j}-\sum_{i \in K} y_{i} \leq b+\bar{u}\left(C^{-}\right)+\gamma
\end{aligned}
$$

is a valid inequality for $P_{N\left(C^{+}\right) \cap F}$.

In the second phase, we lift inequality (14) with $x_{l}, l \in N\left(C^{+}\right) \cap F$. The lifting coefficient of $x_{l}$ equals

$$
\begin{gathered}
g\left(-a_{l}\left(C^{+}\right)\right)=b+\bar{u}\left(C^{-}\right)+\gamma-\max _{(x, y) \in P_{N\left(C^{+}\right) \cap F \backslash, x_{l}=1}}\left\{\sum_{i \in C^{+}} y_{i}+\right. \\
\sum_{j \in N\left(C^{+}\right) \backslash F}\left(a_{j}\left(C^{+}\right)-\lambda+\gamma\right)^{+}\left(1-x_{j}\right)-\sum_{j \in N\left(L^{-}\right) \backslash F} \min \left\{a_{j}\left(L^{-}\right), \lambda-\gamma\right\} x_{j}- \\
\left.\sum_{j \in N\left(L^{-} \cup C^{-}\right) \cap F} \min \left\{a_{j}\left(L^{-} \cup C^{-}\right), \lambda-\gamma\right\} x_{j}-\sum_{i \in K} y_{i}\right\} .
\end{gathered}
$$

The lifting problem has an optimal solution such that $y_{i}=0$ for all $i \in\left(M^{+} \backslash C^{+}\right)$ $\cup K, x_{j}=1$ for all $j \in N\left(C^{+}\right) \backslash F$ such that $a_{j}\left(C^{+}\right) \leq \lambda-\gamma, x_{j}=0$ for all $j \in N\left(L^{-} \cup C^{-}\right) \cap F$ such that $a_{j}\left(L^{-} \cup C^{-}\right) \leq \lambda-\gamma$, and $x_{j}=0$ for all $j \in N\left(L^{-}\right) \backslash F$ such that $a_{j}\left(L^{-}\right) \leq \lambda-\gamma$. Let $(\bar{x}, \bar{y})$ be such an optimal solution. Let $\left.R=\left\{j \in N\left(L^{-} \cup C^{-}\right) \cap F\right): \bar{x}_{j}=1\right\}, S=\left\{j \in N\left(C^{+}\right) \backslash F: \bar{x}_{j}=0\right\}$, and $T=\left\{j \in N\left(L^{-}\right) \backslash F: \bar{x}_{j}=1\right\}$. Again, there are two cases when determining the value of $g\left(-a_{l}\left(C^{+}\right)\right)$depending on how $\sum_{i \in C^{+}} y_{i}$ is bounded in an optimal solution.

$$
\begin{aligned}
\text { Case 1: } \bar{u}\left(C^{+}\right)- & \sum_{j \in S} a_{j}\left(C^{+}\right) \leq b+\bar{u}\left(C^{-}\right)+\gamma+\sum_{j \in R} a_{j}\left(L^{-} \cup C^{-}\right)+\sum_{j \in T} a_{j}\left(L^{-}\right)-a_{l}\left(C^{+}\right) . \\
g\left(-a_{l}\left(C^{+}\right)\right)= & b+\bar{u}\left(C^{-}\right)+\gamma-\left[\bar{u}\left(C^{+}\right)-\right. \\
& \left.\sum_{j \in S} a_{j}\left(C^{+}\right)+a_{l}\left(C^{+}\right)+\sum_{j \in S}\left(a_{j}\left(C^{+}\right)-\lambda+\gamma\right)-\sum_{i \in R \cup T}(\lambda-\gamma)\right] \\
= & (|R \cup S \cup T|-1)(\lambda-\gamma)-a_{l}\left(C^{+}\right) .
\end{aligned}
$$


Case 2: $\bar{u}\left(C^{+}\right)-\sum_{j \in S} a_{j}\left(C^{+}\right)>b+\bar{u}\left(C^{-}\right)+\gamma+\sum_{j \in R} a_{j}\left(L^{-} \cup C^{-}\right)+\sum_{j \in T} a_{j}\left(L^{-}\right)-a_{l}\left(C^{+}\right)$.

$$
\begin{aligned}
g\left(-a_{l}\left(C^{+}\right)\right)= & b+\bar{u}\left(C^{-}\right)+\gamma-\left[b+\bar{u}\left(C^{-}\right)+\gamma+\sum_{j \in R} a_{j}\left(L^{-} \cup C^{-}\right)+\right. \\
& \left.\sum_{j \in T} a_{j}\left(L^{-}\right)+\sum_{j \in S}\left(a_{j}\left(C^{+}\right)-\lambda+\gamma\right)-\sum_{j \in R \cup T}(\lambda-\gamma)\right] \\
= & |R \cup S \cup T|(\lambda-\gamma)-\sum_{j \in S} a_{j}\left(C^{+}\right)-\sum_{j \in R} a_{j}\left(L^{-} \cup C^{-}\right)-\sum_{j \in T} a_{j}\left(L^{-}\right) .
\end{aligned}
$$

Now, let

$$
v_{j}= \begin{cases}a_{j}\left(C^{+}\right), & \text {if } j \in N\left(C^{+}\right) \backslash F, \\ a_{j}\left(L^{-} \cup C^{-}\right), & \text {if } j \in N\left(L^{-} \cup C^{-}\right) \cap F, \\ a_{j}\left(L^{-}\right), & \text {if } j \in N\left(L^{-}\right) \backslash F,\end{cases}
$$

and $\left\{j_{1}, j_{2}, \ldots, j_{r}\right\}=\left\{j \in\left(N\left(C^{+}\right) \backslash F\right) \cup N\left(L^{-}\right) \cup\left(N\left(C^{-}\right) \cap F\right): v_{j}>\lambda-\gamma\right\}$ such that $v_{j_{k}} \geq v_{j_{k+1}}$ for $k=1,2, \ldots r-1$. We also define the partial sums $w_{0}=0$, $w_{k}=\sum_{i=1}^{k} v_{j_{i}}$ for $k=1,2, \ldots, r$.

Next we show that there is a monotone optimal solution to the lifting problem. That is, there exists an optimal solution such that $\min \left\{v_{j}: j \in R \cup S \cup T\right\} \geq \max \left\{v_{j}: j \notin\right.$ $R \cup S \cup T\}$. We prove this claim by a simple interchange argument. For contradiction suppose there is no such solution. Then for some $k \in\{1,2, \ldots r-1\}$ with $v_{j_{k}}>v_{j_{k+1}}$, $j_{k} \notin R \cup S \cup T$ and $j_{k+1} \in R \cup S \cup T$. Let $g^{1}$ be the value of the objective function for this solution. Now, consider the solution obtained by exchanging $j_{k}$ and $j_{k+1}$ and let $g^{2}$ be the corresponding objective function value. If the first solution is in Case 1, i.e., $\lambda-\gamma \leq \sum_{j \in R \cup S \cup T} v_{j}-a_{l}\left(C^{+}\right)$, then $g^{1}=(|R \cup S \cup T|-1)(\lambda-\gamma)-a_{l}\left(C^{+}\right)=g^{2}$. Thus the objective value remains the same. On the other hand, if the first solution is in Case 2, i.e., $\lambda-\gamma>\sum_{j \in R \cup S \cup T} v_{j}-a_{l}\left(C^{+}\right)$, then $g^{1}=|R \cup S \cup T|(\lambda-\gamma)-\sum_{j \in R \cup S \cup T} v_{j}$ and the new solution obtained by the interchange can be in either of the two cases. If the new solution is still in Case 2, then $g^{2}<g^{1}$ since $v_{j_{k}}>v_{j_{k+1}}$. However if the new solution is in Case 1, then $g^{2}=(|R \cup S \cup T|-1)(\lambda-\gamma)-a_{l}\left(C^{+}\right)$. But $g^{2}<g^{1}$ holds again because $\lambda-\gamma>\sum_{j \in R \cup S \cup T} v_{j}-a_{l}\left(C^{+}\right)$. Repeating this interchange whenever a solution is non-monotone, we arrive at a monotone optimal solution, which completes the proof of the claim.

Therefore, we may assume that $R \cup S \cup T$ consists of the first $|R \cup S \cup T|$ elements of $\left\{j_{1}, j_{2}, \ldots, j_{r}\right\}$. Then $g\left(-a_{l}\left(C^{+}\right)\right)$can be expressed in a closed form as follows:

$$
g\left(-a_{l}\left(C^{+}\right)\right)= \begin{cases}k(\lambda-\gamma)-a_{l}\left(C^{+}\right), & w_{k}<a_{l}\left(C^{+}\right) \leq w_{k+1}-\lambda+\gamma, k=0, \ldots, r-1, \\ k(\lambda-\gamma)-w_{k}, & w_{k}-\lambda+\gamma<a_{l}\left(C^{+}\right) \leq w_{k}, k=1, \ldots, r, \\ r(\lambda-\gamma)-w_{r}, & w_{r}<a_{l}\left(C^{+}\right) .\end{cases}
$$

It can be shown that $g$ is superadditive on $\mathbb{R}_{-}$. So from Lemma 1, the lifting is sequence independent and we have the following result. 
Proposition 6. The generalized additive flow cover inequality

$$
\begin{gathered}
\sum_{i \in C^{+}} y_{i}+\sum_{j \in N\left(C^{+}\right) \backslash F}\left(a_{j}\left(C^{+}\right)-\lambda+\gamma\right)^{+}\left(1-x_{j}\right)+ \\
\sum_{j \in N\left(C^{+}\right) \cap F}^{\alpha_{j} x_{j}-} \sum_{j \in N\left(L^{-} \cup C^{-}\right) \cap F} \min \left\{a_{j}\left(L^{-} \cup C^{-}\right), \lambda-\gamma\right\} x_{j}- \\
\sum_{j \in N\left(L^{-}\right) \backslash F} \min \left\{a_{j}\left(L^{-}\right), \lambda-\gamma\right\} x_{j}-\sum_{i \in K} y_{i} \leq b+\bar{u}\left(C^{-}\right)+\gamma
\end{gathered}
$$

with

$$
\alpha_{j}= \begin{cases}k(\lambda-\gamma)-a_{j}\left(C^{+}\right), & w_{k}<a_{j}\left(C^{+}\right) \leq w_{k+1}-\lambda+\gamma, k=0,1, \ldots, r-1, \\ k(\lambda-\gamma)-w_{k}, & w_{k}-\lambda+\gamma<a_{j}\left(C^{+}\right) \leq w_{k}, \quad k=1,2, \ldots, r, \\ r(\lambda-\gamma)-w_{r}, & w_{r}<a_{j}\left(C^{+}\right) .\end{cases}
$$

is valid for $P$, where $w_{0}=0, w_{k}=\sum_{i=1}^{k} v_{j_{i}}$ for $k=1,2, \ldots, r$ and

$$
v_{j}= \begin{cases}a_{j}\left(C^{+}\right), & \text {if } j \in N\left(C^{+}\right) \backslash F, \\ a_{j}\left(L^{-} \cup C^{-}\right), & \text {if } j \in N\left(L^{-} \cup C^{-}\right) \cap F, \\ a_{j}\left(L^{-}\right), & \text {if } j \in N\left(L^{-}\right) \backslash F,\end{cases}
$$

and $\left\{j_{1}, j_{2}, \ldots, j_{r}\right\}=\left\{j \in\left(N\left(C^{+}\right) \backslash F\right) \cup N\left(L^{-}\right) \cup\left(N\left(C^{-}\right) \cap F\right): v_{j}>\lambda-\gamma\right\}$ such that $v_{j_{k}} \geq v_{j_{k+1}}$ for $k=1,2, \ldots, r-1$.

Proposition 7. The generalized additive flow cover inequality (15) is facet-defining for $\operatorname{conv}(P)$ if (12) is facet-defining for $\operatorname{conv}\left(P_{F}\right)$.

\subsection{Generalized additive flow packing inequalities}

Here we generalize the additive flow packing inequalities with the same approach taken in Sect. 2.3 for the additive flow cover inequalities. Consider the projection $P_{F}$ of $P$ introduced in Sect. 2.3. Let $C^{+} \subseteq M^{+}$and $C^{-} \subseteq M^{-}$such that $\mu=$ $b+\bar{u}\left(C^{-}\right)-\bar{u}\left(C^{+}\right)>0$ and $L^{+} \subseteq M^{+} \backslash C^{+}$such that $\gamma=\sum_{i \in L^{+}} u_{i}<\mu$ and $\bar{u}\left(C^{+}\right)+\gamma \geq 0$. Then from Sect. 2.2 we have the following valid additive flow packing inequality for $P_{F}$

$$
\begin{aligned}
& \sum_{i \in C^{+} \cup L^{+}} y_{i}-\sum_{j \in N\left(L^{+}\right) \backslash F} \min \left\{a_{j}\left(L^{+}\right), \mu-\gamma\right\} x_{j}+ \\
& \sum_{j \in N\left(C^{-}\right) \backslash F}\left(a_{j}\left(C^{-}\right)-\mu+\gamma\right)^{+}\left(1-x_{j}\right)-\sum_{i \in K} y_{i} \leq \bar{u}\left(C^{+}\right)+\gamma .
\end{aligned}
$$

To introduce the variables in $F$ into inequality (16), we lift (16) in two phases. First we lift the inequality with the variables in $N\left(C^{+} \cup L^{+}\right) \cap F$. Then in the second phase we lift the resulting inequality with variables in $N\left(C^{-}\right) \cap F$. When employing this two phase lifting procedure, for convenience, we assume that

$$
\text { (A.7) }\left(N\left(L^{+} \cup C^{+}\right) \cap F\right) \cap\left(N\left(C^{-}\right) \cap F\right)=\emptyset \text {. }
$$


The lifting of inequality (16) proceeds similar to the lifting of inequality (12). Therefore, we give only the final result here.

Proposition 8. The generalized additive flow packing inequality

$$
\begin{gathered}
\sum_{i \in C^{+} \cup L^{+}} y_{i}-\sum_{j \in N\left(L^{+}\right) \backslash F} \min \left\{a_{j}\left(L^{+}\right), \mu-\gamma\right\} x_{j}- \\
\sum_{j \in N\left(C^{+} \cup L^{+}\right) \cap F} \min \left\{a_{j}\left(C^{+} \cup L^{+}\right), \mu-\gamma\right\} x_{j}+\sum_{j \in N\left(C^{-}\right) \cap F} \beta_{j} x_{j}- \\
\sum_{j \in N\left(C^{-}\right) \backslash F}\left(a_{j}-\mu+\gamma\right)^{+}\left(1-x_{j}\right)-\sum_{i \in K} y_{i} \leq \bar{u}\left(C^{+}\right)+\gamma
\end{gathered}
$$

with

$$
\beta_{j}= \begin{cases}k(\mu-\gamma)-a_{j}\left(C^{-}\right), & w_{k}<a_{j}\left(C^{-}\right) \leq w_{k+1}-\mu+\gamma, k=0,1, \ldots, r-1, \\ k(\mu-\gamma)-w_{k}, & w_{k}-\mu+\gamma<a_{j}\left(C^{-}\right) \leq w_{k}, \quad k=1,2, \ldots, r, \\ r(\mu-\gamma)-w_{r}, & w_{r}<a_{j}\left(C^{-}\right),\end{cases}
$$

is valid for $P$, where $w_{0}=0, w_{k}=\sum_{i=1}^{k} v_{j_{i}}$ for $k=1,2, \ldots, r$ and

$$
v_{j}= \begin{cases}a_{j}\left(C^{-}\right), & \text {if } j \in N\left(C^{-}\right) \backslash F \\ a_{j}\left(L^{+} \cup C^{+}\right), & \text {if } j \in N\left(L^{+} \cup C^{+}\right) \cap F, \\ a_{j}\left(L^{+}\right), & \text {if } j \in N\left(L^{+}\right) \backslash F\end{cases}
$$

and $\left\{j_{1}, j_{2}, \ldots, j_{r}\right\}=\left\{j \in\left(N\left(C^{-}\right) \backslash F\right) \cup N\left(L^{+}\right) \cup\left(N\left(C^{+}\right) \cap F\right): v_{j}>\mu-\gamma\right\}$ such that $v_{j_{k}} \geq v_{j_{k+1}}$ for $k=1,2, \ldots, r-1$.

Proposition 9. The generalized additive flow packing inequality (17) is facet-defining for $\operatorname{conv}(P)$ if (16) is facet-defining for $\operatorname{conv}\left(P_{F}\right)$.

\subsection{Examples of valid inequalities}

Let $P$ be given by

$$
\begin{aligned}
& y_{1}+y_{2}-y_{3}-y_{4} \leq 2, \\
& y_{1} \leq 4+2 x_{11}+3 x_{12}, y_{2} \leq 3+x_{21}+2 x_{22}, \\
& y_{3} \leq 2+x_{31}+4 x_{32}, y_{4} \leq 4+2 x_{41}+2 x_{42} .
\end{aligned}
$$

The only additive flow cover inequality satisfying Proposition 3 is

$$
y_{1}+\left(1-x_{11}\right)+2\left(1-x_{12}\right)-x_{31}-x_{32}-x_{41}-x_{42} \leq 8,
$$

with $C^{+}=\{1\}$ and $L^{-}=\{3,4\}$, hence $\lambda=7$ and $\gamma=6$. However by projecting a subset $F$ of the binary variables to zero we obtain the following subclass of generalized additive 
flow cover inequalities with $C^{-}=\emptyset$ for $P$ :

\begin{tabular}{c|c|c|l}
$C^{+}$ & $F$ & $L^{-}$ & \multicolumn{1}{|c}{ generalized additive flow cover inequality } \\
\hline 1 & 11 & 4 & $y_{1}-y_{3}-2 x_{11}+2\left(1-x_{12}\right)-x_{41}-x_{42} \leq 6$ \\
2 & 21 & 3 & $y_{2}-y_{4}-x_{21}+\left(1-x_{22}\right)-x_{31}-x_{32} \leq 4$ \\
1,2 & $11,12,21$ & 3,4 & $y_{1}+y_{2}-2 x_{11}-2 x_{12}-x_{21}+\left(1-x_{22}\right)-x_{31}-x_{32}-x_{41}-x_{42} \leq 8$ \\
1,2 & $11,21,22$ & 3,4 & $y_{1}+y_{2}-x_{11}+\left(1-x_{12}\right)-x_{21}-x_{22}-x_{31}-2 x_{32}-2 x_{41}-2 x_{42} \leq 8$ \\
1,2 & $12,21,22$ & 3,4 & $y_{1}+y_{2}+\left(1-x_{11}\right)-2 x_{12}-x_{21}-2 x_{22}-x_{31}-x_{32}-x_{41}-x_{32} \leq 8$
\end{tabular}

By Proposition 7 all of the inequalities given above are facet-defining for $\operatorname{conv}(P)$. Next, we consider the additive flow packing inequalities. The only additive flow packing inequality satisfying Proposition 5 is

$$
y_{1}+y_{2}-y_{4}-2 x_{11}-2 x_{12}-x_{21}-2 x_{22}+2\left(1-x_{32}\right) \leq 7,
$$

with $C^{-}=\{3\}$ and $L^{+}=\{1,2\}$, hence $\mu=9$ and $\gamma=7$. However by projecting a subset of the binary variables $F$ to zero we get the following subclass of generalized additive flow packing inequalities with $C^{+}=\varnothing$ for $P$ :

\begin{tabular}{c|c|c|c}
$C^{-}$ & $F$ & $L^{+}$ & generalized additive flow packing inequality \\
\hline 4 & 41 & 1,2 & $y_{1}+y_{2}-y_{3}-x_{11}-x_{12}-x_{21}-x_{22}-2 x_{41}+\left(1-x_{42}\right) \leq 7$ \\
4 & 42 & 1,2 & $y_{1}+y_{2}-y_{3}-x_{11}-x_{12}-x_{21}-x_{22}+\left(1-x_{41}\right)-2 x_{42} \leq 7$ \\
3 & 31 & 1,2 & $y_{1}+y_{2}-y_{4}-x_{11}-x_{12}-x_{21}-x_{22}-x_{31}+3\left(1-x_{42}\right) \leq 7$
\end{tabular}

By Proposition 9 all of the inequalities given above are facet-defining for $\operatorname{conv}(P)$. Clearly, letting $C^{-}$nonempty for the generalized additive flow cover inequalities, and $\mathrm{C}^{+}$nonempty for the generalized additive flow packing inequalities we get many more valid inequalities for $P$.

\section{Separation}

In this section we discuss how to find valid inequalities that are violated by linear programming solutions. Since the coefficients of the inequalities introduced in Sect. 2 are nonlinear functions of parameters $\lambda, \mu$, and $\gamma$, the separation problems are nonlinear mixed-integer optimization problems. Therefore we take heuristic approaches to solve the related separation problems.

Recall that in the generalized additive flow cover and generalized additive flow packing inequalities, the lifting coefficients of the variables projected to zero $(F)$ are nonpositive. For that reason, in separating these inequalities it is reasonable to let $F$ be the variables for which the LP values are zero. Thus, if $\left(x^{*}, y^{*}\right)$ is the current fractional LP solution, we consider the subclass of the generalized additive flow cover inequalities (15) and generalized additive flow packing inequalities (17) with $F=\left\{j \in N: x_{j}^{*}=0\right\}$. Once a violated additive flow cover or a violated additive flow packing inequality is found in the projected space, we lift it with the variables in $F$. Therefore in the sequel we only discuss the separation of additive flow cover and additive flow packing inequalities. 
The approach we take to separate additive flow cover inequalities is similar to the one given in Nemhauser and Wolsey [11] pp. 499-500 for the flow cover inequalities. In order to find a flow cover, we first assume that $K=\emptyset$, or equivalently $L^{-}=M^{-} \backslash C^{-}$, and relax (7) by reducing $\left(a_{j}\left(C^{+}\right)-\lambda+\gamma\right)^{+}$to $a_{j}\left(C^{+}\right)-\lambda+\gamma$. Then, given a fractional solution $\left(x^{*}, y^{*}\right)$, the violation to maximize is

$$
\sum_{i \in C^{+}} y_{i}^{*}+\sum_{j \in N\left(C^{+}\right)}\left(a_{j}\left(C^{+}\right)-\lambda+\gamma\right)\left(1-x_{j}^{*}\right)-\sum_{j \in N\left(L^{-}\right)}(\lambda-\gamma) x_{j}^{*}-b-u\left(C^{-}\right)-\gamma .
$$

By substituting $u_{i}+\sum_{j \in N(i)} a_{i j} x_{j}^{*}$ for $y_{i}^{*}$ for all $i \in C^{+}$and canceling terms we get the following upper bound on the violation

$$
(\lambda-\gamma)\left(1-\sum_{j \in N\left(C^{+}\right)}\left(1-x_{j}^{*}\right)-\sum_{j \in N\left(L^{-}\right)} x_{j}^{*}\right) .
$$

Now, let $v \in\{0,1\}^{\left|M^{+}\right|}, w \in\{0,1\}^{\left|M^{-}\right|}$be the characteristic vectors of $C^{+}$and $C^{-}$ respectively. The additive flow cover inequality is feasible if $\lambda>\gamma$. This condition can be stated as

$$
\lambda=\sum_{i \in M^{+}} u(i) v_{i}-\sum_{i \in M^{-}} u(i) w_{i}-b>\sum_{i \in M^{-}} u_{i}\left(1-w_{i}\right)=\gamma
$$

Then we have the following 0-1 knapsack problem for finding a cover that maximizes (18)

$$
\begin{gathered}
\min \sum_{i \in M^{+}}\left(\sum_{j \in N(i)}\left(1-x_{j}^{*}\right)\right) v_{i}+\sum_{i \in M^{-}}\left(\sum_{j \in N(i)} x_{j}^{*}\right)\left(1-w_{i}\right) \\
\mathrm{s.t:} \quad \sum_{i \in M^{+}} u(i) v_{i}-\sum_{i \in M^{-}}\left(\sum_{j \in N(i)} a_{i j}\right) w_{i}>b+\sum_{i \in M^{-}} u_{i} \\
v \in\{0,1\}^{\left|M^{+}\right|}, w \in\{0,1\}^{\left|M^{-}\right|} .
\end{gathered}
$$

Note that the step from (18) to (19) is also a heuristic one. If $N(j) \cap N(k) \neq \varnothing$ for $j, k \in C^{+}$or for $j, k \in C^{-}$, then the objective coefficients are larger than the coefficients of (18).

The solution to this knapsack problem gives us a flow cover $\left(C^{+}, C^{-}\right)$. We refer the reader to Martello and Toth [9] for solution methods to solve knapsack problems. In finding this flow cover, we assumed that $L^{-}=M^{-} \backslash C^{-}$. However, it may be possible to increase the violation by considering a subset of $L^{-}$. We also make sure that $u_{i} \geq 0$ for all $i \in L^{-}$to satisfy the facet Condition 4 of Proposition 3. Therefore, we use a greedy heuristic to update $L^{-}$and $K$. Let

$$
\begin{aligned}
l= & \sum_{j \in N\left(C^{+}\right)}\left(a_{j}\left(C^{+}\right)-\lambda+\gamma\right)^{+}\left(1-x_{j}^{*}\right)-\sum_{j \in N\left(L^{-}\right)}(\lambda-\gamma) x_{j}^{*}-\sum_{i \in K} y_{i}^{*}-\mu, \\
l_{k}= & \sum_{j \in N\left(C^{+}\right)}\left(a_{j}\left(C^{+}\right)-\lambda+\gamma-u_{k}\right)^{+}\left(1-x_{j}^{*}\right)-\sum_{j \in N\left(L^{-} \backslash\{k\}\right)}\left(\lambda-\gamma+u_{k}\right) x_{j}^{*}- \\
& \sum_{i \in K \cup\{k\}} y_{i}^{*}-\gamma+u_{k} .
\end{aligned}
$$




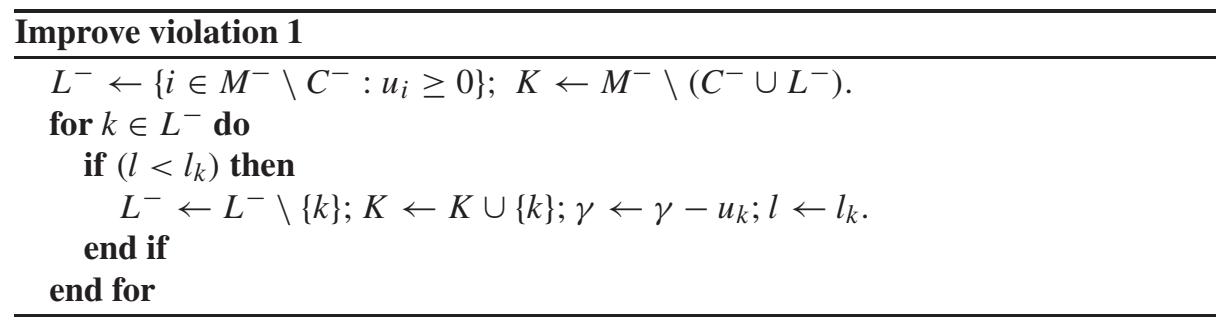

Observe that at the end of the improvement, the resulting inequality is feasible, because for the initial $L^{-}, u_{i} \geq 0$ for all $i \in L^{-}$and therefore $\gamma$ can only decrease, which leaves $\lambda>\gamma$ satisfied. After choosing the sets $C^{+}, C^{-}, L^{-}$, and $K$, we calculate the exact coefficients of the additive flow cover inequality and check for violation. If the inequality is violated by the current fractional solution $\left(x^{*}, y^{*}\right)$, we lift it and add it to the formulation.

Next we discuss how to separate additive flow packing inequalities (10). Since the separation problem is a nonlinear mixed-integer optimization problem, we again take a heuristic approach to find a violated additive flow packing inequality. We first consider the subclass of the inequalities with $L^{+}=M^{+} \backslash C^{+}$. After relaxing the inequality by reducing $\left(a_{j}\left(C^{-}\right)-\mu+\gamma\right)^{+}$to $a_{j}\left(C^{-}\right)-\mu+\gamma$, the violation to maximize is

$$
\sum_{i \in N^{+}} y_{i}^{*}-\sum_{j \in N\left(L^{+}\right)}(\mu-\gamma) x_{j}^{*}+\sum_{j \in N\left(C^{-}\right)}\left(a_{j}\left(C^{-}\right)-\mu+\gamma\right)\left(1-x_{j}^{*}\right)-\sum_{i \in K} y_{i}^{*}-u\left(C^{+}\right)-\gamma .
$$

Since $\sum_{i \in N^{+}} y_{i}^{*} \leq b+\sum_{i \in K} y_{i}^{*}+\sum_{i \in C^{-}}\left(u_{i}+\sum_{j \in N(i)} a_{i j} x_{j}^{*}\right)$, we see that the violation is bounded above by

$$
(\mu-\gamma)\left(1-\sum_{j \in N\left(L^{+}\right)} x_{j}^{*}-\sum_{j \in N\left(C^{-}\right)}\left(1-x_{j}^{*}\right)\right) .
$$

Using the same definition for $v$ and $w$, since $\mu>\gamma$ can be written as

$$
\mu=b+\sum_{i \in M^{-}} u(i) w_{i}-\sum_{i \in M^{+}} u(i) v_{i}>\sum_{i \in M^{+}} u_{i}\left(1-v_{i}\right)=\gamma
$$

we get the following 0-1 knapsack problem to find a violated additive flow packing inequality

$$
\begin{gathered}
\min \sum_{i \in M^{+}}\left(\sum_{j \in N(i)} x_{j}^{*}\right)\left(1-v_{i}\right)+\sum_{i \in M^{-}}\left(\sum_{j \in N(i)}\left(1-x_{j}^{*}\right)\right) w_{i} \\
\text { s.t: } \quad \sum_{i \in M^{+}}\left(\sum_{j \in N(i)} a_{i j}\right) v_{i}-\sum_{i \in M^{-}} u(i) w_{i}<b-\sum_{i \in M^{+}} u_{i} \\
v \in\{0,1\}^{\left|M^{+}\right|}, w \in\{0,1\}^{\left|M^{-}\right|} .
\end{gathered}
$$

Note again that the step from (20) to (21) is also a heuristic one. If $N(j) \cap N(k) \neq \emptyset$ for $j, k \in C^{+}$or for $j, k \in C^{-}$, then the objective coefficients are larger than the coefficients of inequality (20). Once a flow packing is found in this way, we employ a violation improvement heuristic similar to the one given for the additive flow cover inequalities. If the inequality is violated by the LP solution, we lift it and add it to the formulation. 


\section{Computational results}

In this section, we present our computational results on solving network expansion problems with a branch-and-cut algorithm. We implemented heuristic separation algorithms for the generalized additive flow cover and flow packing inequalities for the single node relaxation of the problem. We also used the lifted cover inequalities [6] for surrogate $0-1$ knapsack relaxations of the single node relaxation, where the continuous flow variables are replaced with either their 0-1 additive variable upper bound variables or with their lower bounds. The branch-and-cut algorithm was implemented with MINTO [10] (version 3.0) using CPLEX as LP solver (version 6.0). All of the experiments were performed on a SUN Ultra 10 workstation with a one hour CPU time limit and a 100,000 nodes search tree size limit.

We present a summary of two experiments. Our data set consists of randomly generated network expansion problems with step function capacity installation costs, which are available at http://ieor.berkeley.edu/ atamturk/data/avub. In the formulation of these problems, for each arc $i$ we have two constraints

$$
\begin{gathered}
y_{i} \leq u_{i}+\sum_{j=1}^{k} a_{i j} x_{i j}, \\
\sum_{j=1}^{k} x_{i j} \leq 1,
\end{gathered}
$$

where $a_{i j-1}<a_{i j}$ for $2 \leq j \leq k$. In the data set $50 \%$ of the nodes are demand nodes and $20 \%$ percent of the nodes are supply nodes. Demand is uniformly distributed between 1 and 10. We let $a_{i 1}=t d /(n s \times k)$, where $t d$ is total demand and is $n s$ the number of supply nodes and $a_{i j}=2.25 \times a_{i j-1}$ for $2 \leq j \leq k$. To introduce an economy of scale to the costs, we let the cost for installing capacity $a_{i j}, c_{i j}=0.75 \times 2.25 \times c_{i j-1}$ for $2 \leq j \leq k$ and $c_{i 1}=1$.

The first experiment is performed to test the effectiveness of the cuts in solving problems with 20 vertices and $70 \%$ edge density. These problems were solved using MINTO first with its default settings and then with the above mentioned cutting planes generated throughout the search tree. In Table 1, we report the number of AVUB variables per flow variable $(a v u b s=k$ ) and the average values for the LP relaxation at the root node of the search tree (zroot), the best lower bound (zlb) and the best upper bound (zub) on the optimal value at termination, the percentage gap between $z l b$ and zub (endgap), the number of generalized additive flow cover cuts (gafcov), generalized additive flow packing cuts (gafpack), surrogate knapsack cover cuts (skcov) added, the number of nodes evaluated (nodes), and the CPU time elapsed in seconds ( $t$ ime) for five random instances. While none of the problems could be solved to optimality without adding the cuts within 100,000 nodes, all of the problems were solved easily when the cuts were added. We note that MINTO does not generate any system cuts for these problems, since it does not recognize that additive variable upper bounds can be relaxed to simple variable upper bounds. Observe that the addition of the cuts improves the lower bounds as well as the upper bounds significantly, which leads to much smaller 
search trees and overall solution times. Table 1 clearly demonstrates the effectiveness of the cuts.

Table 1. Effectiveness of cuts: 20 vertices

\begin{tabular}{l|c|rrrrrrrrr}
\hline & avubs & zroot & zlb & zub & endgap & gafcov & gafpack & skcov & nodes & time \\
\hline \multirow{2}{*}{ without } & 2 & 9.49 & 10.22 & 16.80 & 39.00 & 0 & 0 & 0 & 100,000 & 1386 \\
cuts & 4 & 12.74 & 16.99 & 25.60 & 33.86 & 0 & 0 & 0 & 100,000 & 1018 \\
& 8 & 2.77 & 11.51 & 58.40 & 79.15 & 0 & 0 & 0 & 100,000 & 1859 \\
\hline \multirow{2}{*}{ with } & 2 & 15.30 & 15.40 & 15.40 & 0.00 & 46 & 42 & 15 & 6 & 1 \\
cuts & 4 & 23.73 & 25.20 & 25.20 & 0.00 & 55 & 33 & 47 & 73 \\
& 8 & 36.36 & 38.40 & 38.40 & 0.00 & 47 & 34 & 41 & 121 & 9 \\
\hline
\end{tabular}

In the next experiment, we solved larger instances of the network expansion problem with $20 \%$ edge density to find out the sizes of instances that can be solved with the branch-and-cut algorithm. The results of this experiment are summarized in Table 2, where we present the number of AVUB variables per flow variable (avubs), the average values for the percentage difference between the initial LP relaxation and zub (initgap), the percentage difference between the LP relaxation after the cuts are added at the root node and zub (rootgap), in addition to endgap, gafcov, gafpack, skcov, nodes, and time for five random instances with 50, 100 and 150 vertices. We note that these problems are much larger than ones for which computations are provided in the literature $[5,14]$. Although all of the instances with 50 vertices could be solved to optimality, for the larger instances the gap between the best lower bound and the best upper bound could not be completely closed for most of the problems with 4 or 8 avubs within an hour of CPU time. Nevertheless, the improvement in LP relaxations is very good, ranging between $50 \%$ and $98 \%$.

Table 2. Performance of the branch-and-cut algorithm

\begin{tabular}{c|c|rrrrrrrr}
\hline vertices & avubs & initgap & rootgap & endgap & gafcov & gafpack & skcov & nodes & time \\
\hline \multirow{5}{*}{50} & 1 & 25.09 & 1.05 & 0.00 & 48 & 56 & 16 & 24 & 2 \\
& 2 & 20.12 & 2.20 & 0.00 & 115 & 102 & 38 & 110 & 11 \\
& 4 & 36.34 & 3.31 & 0.00 & 192 & 139 & 106 & 721 & 49 \\
& 8 & 92.06 & 3.72 & 0.00 & 416 & 216 & 164 & 7712 & 960 \\
\hline \multirow{4}{*}{100} & 1 & 14.80 & 0.94 & 0.00 & 182 & 148 & 41 & 44 & 39 \\
& 2 & 12.27 & 4.44 & 0.00 & 590 & 324 & 191 & 1236 & 961 \\
& 4 & 38.04 & 3.74 & 2.37 & 707 & 382 & 448 & 2437 & 2171 \\
& 8 & 92.75 & 9.15 & 8.75 & 828 & 408 & 509 & 1717 & 3600 \\
\hline \multirow{4}{*}{150} & 1 & 16.56 & 0.29 & 0.00 & 480 & 307 & 57 & 346 & 586 \\
& 2 & 10.81 & 5.39 & 4.34 & 586 & 395 & 318 & 813 & 2965 \\
& 4 & 43.69 & 12.22 & 12.22 & 862 & 527 & 672 & 711 & 3600 \\
& 8 & 93.08 & 15.02 & 15.02 & 659 & 449 & 503 & 477 & 3600 \\
\hline
\end{tabular}

For most of the unsolved problems, the best lower bound and the best upper bound were found at the root node in a few minutes; no improvement in the gap was observed later in the search tree. For instance nexp.100.8.5 the value of the initial LP relaxation was 18.23. After adding 916 cuts in 42 rounds the root LP relaxation improved to 220.81 , which was in fact the best lower bound found in the search tree, in 617 seconds. 
The best upper bound 259 was also found at the root node by a simple heuristic which installs the least cost integral capacity feasible for the flow on each edge provided by the LP relaxation. Therefore, for the unsolved problems it is likely that the actual duality gaps of the improved LP relaxations are much smaller.

More detailed experiments to compare the relative effectiveness of the different classes of cuts revealed that the generalized additive flow cover inequalities were the most effective, and that the lifted surrogate knapsack inequalities were more effective than the generalized additive flow packing inequalities. However, the use of all three classes of cuts delivered the best performance in most cases. From these computational results, we conclude that the valid inequalities derived from the single node relaxations are very effective in improving the LP bounds for network expansion problems.

\section{References}

1. Alevras, D., Grötschel, M., Wessäly, R. (1998): Cost-efficient network synthesis from leased lines. Annals of Operations Research 76, 1-20

2. Atamtürk, A. (1998): Conflict graphs and flow models for mixed-integer linear optimization problems. $\mathrm{PhD}$ thesis, ISyE, Georgia Institute of Technology, Atlanta, USA

3. Atamtürk, A. (1999): Lifted flow pack facets of the single node fixed-charge flow polytope. Research report, IEOR, University of California at Berkeley. Available at http: / / ieor. berkeley . edu/ atamturk

4. Balakrishnan, A., Magnanti, T.L., Shulman, A., Wong, R.T. (1991): Models for planning capacity expansion in local access telecommunication networks. Annals of Operations Research 33, 239-284

5. Bienstock, D., Günlük, O. (1996): Capacitated network design - Polyhedral structure and computation. INFORMS Journal on Computing 8, 243-259

6. Gu, Z., Nemhauser, G.L., Savelsbergh, M.W.P. (1998): Lifted covers inequalities for 0-1 integer programs: Computation. INFORMS Journal on Computing 10, 427-437

7. Gu, Z., Nemhauser, G.L., Savelsbergh, M.W.P. (1999): Lifted flow cover inequalities for mixed 0-1 integer programs. Mathematical Programming 85, 439-467

8. Gu, Z., Nemhauser, G.L., Savelsbergh, M.W.P. (2000): Sequence independent lifting in mixed integer programming. Journal of Combinatorial Optimization 4, 109-129

9. Martello, S., Toth, P. (1990): Knapsack Problems: Algorithms and Computer Implementations. John Wiley and Sons, Inc, New York

10. Nemhauser, G.L., Savelsbergh, M.W.P., Sigismondi, G.S. (1994): MINTO, a Mixed INTeger Optimizer. Operations Research Letters 15, 47-58

11. Nemhauser, G.L., Wolsey, L.A. (1988): Integer and Combinatorial Optimization. John Wiley and Sons, New York

12. Padberg, M.W., Van Roy, T.J., Wolsey, L.A. (1984): Valid linear inequalities for fixed charge problems. Operations Research 32, 842-861

13. Stallaert, J.I.A. (1997): The complementary class of generalized flow cover inequalities. Discrete Applied Mathematics 77, 73-80

14. Stoer, M., Dahl, G. (1994): A polyhedral approach to multicommodity survivable network. Numerische Mathematik 68, 149-167

15. Van Roy, T.J., Wolsey, L.A. (1986): Valid inequalities for mixed 0-1 programs. Discrete Applied Mathematics 14, 199-213

16. Wolsey, L.A. (1977): Valid inequalities and superadditivity for 0/1 integer programs. Mathematics of Operations Research 2, 66-77 